\title{
Lie Symmetry and the Bethe Ansatz Solution of a New Quasi-Exactly Solvable Double-Well Potential
}

\author{
M. Baradaran and H. Panahi \\ Department of Physics, University of Guilan, Rasht 41635-1914, Iran \\ Correspondence should be addressed to H. Panahi; t-panahi@guilan.ac.ir
}

Received 4 November 2016; Revised 14 January 2017; Accepted 24 January 2017; Published 2 March 2017

Academic Editor: Akpan Ikot

Copyright ( 2017 M. Baradaran and H. Panahi. This is an open access article distributed under the Creative Commons Attribution License, which permits unrestricted use, distribution, and reproduction in any medium, provided the original work is properly cited. The publication of this article was funded by $\mathrm{SCOAP}^{3}$.

\begin{abstract}
We study the Schrödinger equation with a new quasi-exactly solvable double-well potential. Exact expressions for the energies, the corresponding wave functions, and the allowed values of the potential parameters are obtained using two different methods, the Bethe ansatz method and the Lie algebraic approach. Some numerical results are reported and it is shown that the results are in good agreement with each other and with those obtained previously via a different method.
\end{abstract}

\section{Introduction}

A quantum mechanical system is exactly solvable (ES) if all the eigenvalues and corresponding eigenfunctions can be determined exactly through algebraic means. These quantum systems play an important role in various branches of physics. However, such systems are rare and the Schrödinger equation cannot be solved exactly to obtain the whole spectrum except for a limited number of potentials, such as the harmonic oscillator and Coulomb and Pöschl-Teller potentials [1-5]. A review of early works in this area can be found in [68]. In contrast, a quantum system is called quasi-exactly solvable (QES) if only a finite part of the spectrum can be found exactly [9-12]. During the last decades, the QES models have received a great deal of attention because of their wide applications in quantum mechanics [13-21]. These models are distinguished by the fact that their infinitedimensional Hamiltonian can be reduced to a block diagonal matrix with at least a finite-dimensional block, in which its eigenvalues and eigenfunctions can always be determined by diagonalizing the corresponding matrix. On the other hand, during the last decades, a great deal of attention has been given to the study of the Schrödinger equation with QES double-well potential (DWP) including the quartic potential [22], the sextic potential [23], and the Razavy potential [24]. In the literature, there are two distinct approaches for investigating the QES systems: the Lie algebraic approach [9$11]$ and the analytical approach $[12,15]$ which is based on the Bethe ansatz method (BAM). The interested reader is referred to $[4,25-29]$ and references therein for more detailed information regarding the application of the wave function ansatz method in physical problems. In this paper, applying the analytical approach of quasi-exact solvability, we investigate the Schrödinger equation for a new type of one-dimensional QES DWP proposed by Chen et al. [17]. They studied the problem and obtained solutions of the first two states by using two methods, the confluent Heun functions and the Wronskian method [17]. Within the present study, through the BAM, we are going to extend the results of [17] by finding general exact expressions for the energies, the wave functions, and the special constraints on the potential parameters. Also, we solve the same problem using the Lie algebraic approach and illustrate how the relation with $s l(2)$ Lie algebra underlies the quasi-exact solvability of the DWP model; also we show that the results of the two methods are consistent.

This paper is organized as follows: In Section 2, we introduce the QES DWP and solve the corresponding Schrödinger equation using the BAM. Also, the closed form expressions for the energies and the wave functions are obtained in terms of the roots of the Bethe ansatz equations. In Section 3, we solve the same problems using the Lie algebraic approach and demonstrate that the system possesses a hidden $\operatorname{sl}(2)$ 
algebraic symmetry which is responsible for quasi-exact solvability. Some numerical results, obtained by the BAM and QES methods, are reported and discussed in Section 4. Finally, in Section 5, we present the conclusions.

\section{The Analytical Method Based on the Bethe Ansatz for the QES DWP}

We begin with the one-dimensional three-parameter QES DWP proposed by Chen et al. [17] as

$$
\begin{aligned}
V(x)= & \frac{v_{1}}{\cosh ^{2}(x)}+\frac{v_{2}}{1+g \cosh ^{2}(x)} \\
& +\frac{v_{3}}{\left(1+g \cosh ^{2}(x)\right)^{2}},
\end{aligned}
$$

which, under the condition $g \gg 1$, becomes the Manning potential [30]

$$
\begin{aligned}
V_{\text {Manning }}(x) & =v_{4} \operatorname{sech}^{2}(x)+v_{5} \operatorname{sech}^{4}(x), \\
v_{4} & =v_{1}+\frac{v_{2}}{g}, \\
v_{5} & =\frac{v_{3}}{g^{2}} .
\end{aligned}
$$

In 1935, Manning used this symmetric double minima potential to study the vibrational normal modes of the ND3 and NH3 molecules [30]. This application is possible because the nitrogen atom in these molecules has two equilibrium positions on either side of the D2 and $H_{2}$ planes. In the following, we show that under certain constraints on the potential parameters $v_{1}, v_{2}$, and $v_{3}$, a finite number of the energy eigenvalues and eigenfunctions of the corresponding Schrödinger operator can be obtained exactly in explicit form. In Figure 2, we draw potential (1) for the allowed values of potential parameters. In atomic units $(m=\hbar=c=1)$, the Schrödinger equation with potential (1) is written as

$$
\begin{gathered}
\left(-\frac{d^{2}}{d x^{2}}+\frac{v_{1}}{\cosh ^{2}(x)}+\frac{v_{2}}{1+g \cosh ^{2}(x)}\right. \\
\left.+\frac{v_{3}}{\left(1+g \cosh ^{2}(x)\right)^{2}}\right) \psi(x)=E \psi(x) .
\end{gathered}
$$

Chen et al. [17] studied this problem earlier and presented the results of the first two states in terms of confluent Heun functions. Here, we intend to extend their results by calculating the general exact solutions of the problem in terms of the roots of a set of algebraic equations within the framework of the Bethe ansatz. For this aim, making the change of variable $z=-\sinh ^{2}(x)$ and taking the ansatz for the wave function

$$
\begin{aligned}
\psi(x)= & (\cosh (x))^{(1 / 2)\left(1+\sqrt{1-4 v_{1}}\right)} \\
& \cdot\left(1+g \cosh ^{2}(x)\right)^{(1 / 2)\left(1-\sqrt{1+v_{3} /(1+g)}\right)} \phi(z),
\end{aligned}
$$

we obtain

$$
H \phi(z)=0 \text {, }
$$

$H$

$$
\begin{aligned}
= & \left(z^{3}-(\lambda+1) z^{2}+\lambda z\right) \frac{d^{2}}{d z^{2}} \\
& +\left((\gamma+\delta+\varepsilon) z^{2}-(\lambda \gamma+\gamma+\lambda \delta+\varepsilon) z+\lambda \gamma\right) \frac{d}{d z} \\
& +(\alpha \beta z-\sigma),
\end{aligned}
$$

$$
\begin{aligned}
\lambda= & \frac{1+g}{g}, \\
\sigma= & \frac{\lambda}{4}\left(\frac{1}{2}+\frac{1}{2} \sqrt{1-4 v_{1}}+\frac{1}{\lambda}-\frac{1}{\lambda} \sqrt{1+\frac{v_{3}}{1+g}}-v_{1}\right. \\
& \left.-\frac{v_{2}}{g \lambda}-\frac{v_{3}}{g^{2} \lambda^{2}}+E\right), \\
\varepsilon & =\alpha+\beta+1-\gamma-\delta .
\end{aligned}
$$

In order to apply the BAM to the present problem, we suppose that (5) has degree $n$ polynomial solution as (the Bethe ansatz)

$$
\phi_{n}(z)= \begin{cases}\prod_{k=1}^{n}\left(z-z_{k}\right) & n \neq 0 \\ 1 & n=0\end{cases}
$$

with undetermined, distinct roots $z_{k}$ which are none other than the wave function nodes. Substituting (7) into (5) and after some algebra, we obtain

$$
\begin{array}{r}
\frac{\lambda}{4}\left(\frac{1}{2}+\frac{1}{2} \sqrt{1-4 v_{1}}+\frac{1}{\lambda}-\frac{1}{\lambda} \sqrt{1+\frac{v_{3}}{1+g}}-v_{1}-\frac{v_{2}}{g \lambda}\right. \\
\left.-\frac{v_{3}}{g^{2} \lambda^{2}}+E\right)=(n(n-1)+n(\gamma+\delta+\varepsilon)+\alpha \beta) z \\
+\sum_{k=1}^{n} \frac{1}{z-z_{k}}\left(\left(z_{k}^{3}-(\lambda+1) z_{k}^{2}+\lambda z_{k}\right) \sum_{j \neq k}^{n} \frac{2}{z_{k}-z_{j}}\right. \\
\left.+\left((\gamma+\delta+\varepsilon) z_{k}^{2}-(\lambda \gamma+\gamma+\lambda \delta+\varepsilon) z_{k}+\lambda \gamma\right)\right)
\end{array}
$$




$$
\begin{aligned}
& +(2(n-1)+\gamma+\delta+\varepsilon) \sum_{k=1}^{n} z_{k}-n(n-1)(\lambda+1) \\
& -n(\lambda \gamma+\gamma+\lambda \delta+\varepsilon)
\end{aligned}
$$

Comparing the left and right hand sides of (8), we obtain the following relations:

$$
\begin{aligned}
& -\sum_{j \neq k}^{n} \frac{2}{z_{k}-z_{j}}-\frac{\gamma}{z_{k}}-\frac{\delta}{z_{k}-1}-\frac{\varepsilon}{z_{k}-\lambda}=0, \\
& \frac{1}{16}\left(3+\sqrt{1-4 v_{1}}-2 \sqrt{1+\frac{v_{3}}{1+g}}\right)^{2}+\frac{E}{4}=-n(n \\
& \quad-1)-n(\gamma+\delta+\varepsilon), \\
& -\left(\frac { \lambda } { 4 } \left(\frac{1}{2}+\frac{1}{2} \sqrt{1-4 v_{1}}+\frac{1}{\lambda}-\frac{1}{\lambda} \sqrt{1+\frac{v_{3}}{1+g}}-v_{1}\right.\right. \\
& \left.\left.\quad-\frac{v_{2}}{g \lambda}-\frac{v_{3}}{g^{2} \lambda^{2}}+E\right)\right)=-(2(n-1)+\gamma+\delta+\varepsilon) \\
& \quad \cdot \sum_{k=1}^{n} z_{k}+n(n-1)(\lambda+1)+n(\lambda \gamma+\gamma+\lambda \delta+\varepsilon),
\end{aligned}
$$

for the roots $z_{k}$ (the so-called Bethe ansatz equation), the energy eigenvalues, and the restrictions on the parameters of the potential, respectively. Now, let us look at Bethe ansatz equations (9) from a different point of view. It is not difficult to understand that the set of algebraic equations (9) can be interpreted as the equilibrium condition of an analogue mechanical system consisting of $n$ particles with coordinates $z_{k}$ moving in the interaction potential

$$
\begin{aligned}
U\left(z_{1}, \ldots, z_{n}\right) \\
=-2 \sum_{j \neq k}^{n} \ln \left|z_{k}-z_{j}\right| \\
\quad-\sum_{k=1}^{n}\left(\gamma \ln z_{k}+\delta \ln \left(z_{k}-1\right)+\varepsilon \ln \left(z_{k}-\lambda\right)\right) .
\end{aligned}
$$

In other words, the $n$ equations $\partial U / \partial z_{k}(k=1,2, \ldots, n)$ describing the equilibrium distribution of the particles coincide with (9). The first term in this expression is none other than the Coulomb repulsion of the particles while the three terms in the parenthesis correspond to the interactions of each individual particle with the force centers located at the origin, 1 and $\lambda$, respectively. Therefore, from the point of view of classical mechanics, the problem of finding the roots of the set of algebraic Bethe ansatz equations (9) is equivalent to the problem of finding equilibrium positions of a system of classical Coulomb particles in the interaction potential $U\left(z_{1}, \ldots, z_{n}\right)$. In the following, to clarify how the method works, we study the ground and the first and the second excited states of the model in more detail. For $n=0$, from (10) and (4), we have the following relations:

$$
\begin{aligned}
& \frac{1}{16}\left(3+\sqrt{1-4 v_{1}}-2 \sqrt{1+\frac{v_{3}}{1+g}}\right)^{2}+\frac{E}{4}=0, \\
& \psi_{0}(x)=(\cosh (x))^{(1 / 2)\left(1+\sqrt{1-4 v_{1}}\right)} \\
& \cdot\left(1+g \cosh ^{2}(x)\right)^{(1 / 2)\left(1-\sqrt{1+v_{3} /(1+g)}\right)},
\end{aligned}
$$

for the ground state energy and the corresponding wave function, where the potential parameters from (11) satisfy

$$
\begin{aligned}
& \frac{\lambda}{4}\left(\frac{1}{2}+\frac{1}{2} \sqrt{1-4 v_{1}}+\frac{1}{\lambda}-\frac{1}{\lambda} \sqrt{1+\frac{v_{3}}{1+g}}-v_{1}-\frac{v_{2}}{g \lambda}\right. \\
& \left.-\frac{v_{3}}{g^{2} \lambda^{2}}+E\right)=0 .
\end{aligned}
$$

For $n=1$, by (10) and (4), we obtain the first excited state energy and wave function as

$$
\begin{aligned}
& \frac{1}{16}\left(3+\sqrt{1-4 v_{1}}-2 \sqrt{1+\frac{v_{3}}{1+g}}\right)^{2}+\frac{E}{4} \\
& =-(\gamma+\delta+\varepsilon) \\
& \psi_{1}(x)=(\cosh (x))^{(1 / 2)\left(1+\sqrt{1-4 v_{1}}\right)} \\
& \cdot\left(1+g \cosh ^{2}(x)\right)^{(1 / 2)\left(1-\sqrt{1+v_{3} /(1+g)}\right)} \\
& \cdot\left(-\sinh ^{2}(x)-z_{1}\right)
\end{aligned}
$$

respectively. In this case, the constraint on the potential parameters is given by

$$
\begin{aligned}
& -\frac{\lambda}{4}\left(\frac{1}{2}+\frac{1}{2} \sqrt{1-4 v_{1}}+\frac{1}{\lambda}-\frac{1}{\lambda} \sqrt{1+\frac{v_{3}}{1+g}}-v_{1}-\frac{v_{2}}{g \lambda}\right. \\
& \left.-\frac{v_{3}}{g^{2} \lambda^{2}}+E\right)+(\gamma+\delta+\varepsilon) z_{1}+(\lambda \gamma+\gamma+\lambda \delta+\varepsilon) \\
& =0
\end{aligned}
$$

where the root $z_{1}$ of the wave function is obtainable from Bethe ansatz equation (9) as

$$
z_{1}=\frac{\lambda \delta+\lambda \gamma+\gamma+\epsilon \pm \sqrt{(\lambda-1)^{2} \gamma^{2}+2(\lambda-1)(\lambda \delta-\epsilon) \gamma+(\lambda \delta+\epsilon)^{2}}}{2 \gamma+2 \delta+2 \epsilon}
$$


Analogously, for the second excited state $n=2$, we have

$$
\begin{aligned}
& \frac{1}{16}\left(3+\sqrt{1-4 v_{1}}-2 \sqrt{1+\frac{v_{3}}{1+g}}\right)^{2}+\frac{E}{4}=-2 \\
& \quad-2(\gamma+\delta+\varepsilon), \\
& \psi_{2}(x)=(\cosh (x))^{(1 / 2)\left(1+\sqrt{1-4 v_{1}}\right)} \\
& \quad \cdot\left(1+g \cosh ^{2}(x)\right)^{(1 / 2)\left(1-\sqrt{1+v_{3} /(1+g)}\right)} \\
& \quad \times\left(\sinh ^{4}(x)+\left(z_{1}+z_{2}\right) \sinh ^{2}(x)+z_{1} z_{2}\right),
\end{aligned}
$$

$$
\left(z_{1}, z_{2}\right)=\left\{\begin{array}{l}
(9.714045208,0.2859547921) \\
(0.6953879418,0.1092848103) \\
(7.229242571-4.010375187 i, 7.229242571+4.010375187 i)
\end{array}\right.
$$

Here, we have taken the parameters $v_{1}=0.09, v_{3}=10$, and $g=1 / 4$. In Table 1 , we have reported and compared our numerical results for the first four states. As an additional comment on the treatment of the wave functions, from (13), (15), and (18) it can be seen that except the ground state wave function, the higher excited states are mathematically meaningless for large $x$, since, as $x \rightarrow \pm \infty$, we have $|z|=$ $\sinh ^{2}(x) \rightarrow \infty$. This property can be explained well by the asymptotic behavior of the Heun function which is only convergent within the circle $|z|<1$. The interested reader is referred to [17] for details of the problem.

\section{The Lie Algebraic Approach for the QES DWP}

In the previous section, we have investigated the Schrödinger equation for the QES DWP and obtained the general exact solutions of the system within the framework of the Bethe ansatz. In this section, we solve the same problem by using the Lie algebraic approach of quasi-exact solvability and obtain the exact solutions through the $s l(2)$ algebraization. A differential equation is said to be QES if it is an element of the universal enveloping algebra of a finite-dimensional QES Lie algebra of differential operators [10]. In one dimension, the Lie algebra $s l(2)$ is the only algebra of differential operators with finite-dimensional representations [11]. The usual realization of the $s l(2)$ Lie algebra is given by the following differential operators $[10,11]$ :

$$
\begin{aligned}
& J_{n}^{+}=-z^{2} \frac{d}{d z}+n z, \\
& J_{n}^{0}=z \frac{d}{d z}-\frac{n}{2}, \\
& J_{n}^{-}=\frac{d}{d z},
\end{aligned}
$$

for the energy and wave function, respectively, where the potential parameters satisfy the constraint condition

$$
\begin{aligned}
& -\frac{\lambda}{4}\left(\frac{1}{2}+\frac{1}{2} \sqrt{1-4 v_{1}}+\frac{1}{\lambda}-\frac{1}{\lambda} \sqrt{1+\frac{v_{3}}{1+g}}-v_{1}-\frac{v_{2}}{g \lambda}\right. \\
& \left.-\frac{v_{3}}{g^{2} \lambda^{2}}+E\right)+(2+\gamma+\delta+\varepsilon)\left(z_{1}+z_{2}\right)+2(\lambda \\
& +1)+2(\lambda \gamma+\gamma+\lambda \delta+\varepsilon)=0,
\end{aligned}
$$

and the roots $z_{1}$ and $z_{2}$ are obtainable from Bethe ansatz equations (9) as

which leave the finite-dimensional space invariant:

$$
P_{n+1}=\left\langle 1, z, z^{2}, \ldots, z^{n}\right\rangle
$$

Therefore, the most general one-dimensional second-order QES differential equation can be expressed as a quadratic combination of the $s l(2)$ generators as

$$
H=\sum_{a, b=0, \pm} C_{a b} J_{n}^{a} J_{n}^{b}+\sum_{a=0, \pm} C_{a} J_{n}^{a}+C, \quad C, C_{a}, C_{a b} \in \mathbb{R}
$$

which clearly preserves $(n+1)$-dimensional representation space (22). In general, by means of appropriate transformations, the operator $H$ can always be reduced to a Schrödingertype operator. Conversely, it is not difficult to verify that (5) can be expressed as a special case of the Lie algebraic form (23) as

$$
\begin{aligned}
& H \phi(z)=0, \\
& \begin{aligned}
& H \\
&=- J_{n}^{+} J_{n}^{0}+(\lambda+1) J_{n}^{+} J_{n}^{-}+a J_{n}^{0} J_{n}^{-} \\
&+\left(\left(1-\frac{3 n}{2}\right)-(\gamma+\delta+\varepsilon)\right) J_{n}^{+} \\
&-((\lambda \gamma+\gamma+\lambda \delta+\varepsilon)+n(\lambda+1)) J_{n}^{0} \\
&+\left(\lambda \gamma+\frac{n \lambda}{2}\right) J_{n}^{-} \\
&+\left(-\sigma-\frac{n(\lambda \gamma+\gamma+\lambda \delta+\varepsilon)}{2}-\frac{n^{2}(\lambda+1)}{2}\right),
\end{aligned}
\end{aligned}
$$


TABLE 1: Solutions of the first four states for the QES DWP with $v_{1}=0.09, v_{3}=10$, and $g=1 / 4$.

\begin{tabular}{|c|c|c|c|c|c|c|}
\hline$n$ & $\begin{array}{l}\text { Energy } \\
\text { (BAM) } \\
\text { Eq. (10) }\end{array}$ & $\begin{array}{c}\text { Energy } \\
\text { (QES) } \\
\text { Eq. (25) }\end{array}$ & $\begin{array}{l}\text { Energy } \\
\text { Ref. [17] }\end{array}$ & $\begin{array}{c}v_{2} \\
\text { (BAM) } \\
\text { Eq. (11) }\end{array}$ & $\begin{array}{c}v_{2} \\
(\mathrm{QES}) \\
\text { Eq. }(26)\end{array}$ & $\begin{array}{c}\quad v_{2} \\
\text { Ref. [17] }\end{array}$ \\
\hline 0 & -1.21 & -1.21 & -1.21 & -9 & -9 & -9 \\
\hline 1 & -0.81 & -0.81 & - & $\begin{array}{r}-8.531128900 \\
-0.4688711260\end{array}$ & $\begin{array}{l}-8.531128874 \\
-0.4688711258\end{array}$ & - \\
\hline 2 & -8.41 & -8.41 & - & $\begin{array}{c}-9 \\
8.471121770 \\
-17.47112177\end{array}$ & $\begin{array}{c}-9 \\
8.471121770 \\
-17.47112177\end{array}$ & - \\
\hline 3 & -24.01 & -24.01 & - & $\begin{array}{c}-9 \\
-26.41460699 \\
17.41460700\end{array}$ & $\begin{array}{c}-9 \\
-36 \\
-26.41460700 \\
17.41460700\end{array}$ & - \\
\hline
\end{tabular}

if the following constraint (the condition of quasi-exact solvability) is satisfied

$$
\begin{aligned}
& \frac{1}{16}\left(3+\sqrt{1-4 v_{1}}-2 \sqrt{1+\frac{v_{3}}{1+g}}\right)^{2}+\frac{E}{4} \\
& \quad=n-n(\gamma+\delta+\varepsilon)-n^{2} .
\end{aligned}
$$

$$
\left(\begin{array}{cccccc}
-\sigma & \lambda \gamma & 0 & 0 & 0 & 0 \\
\left(n-n^{2}\right)-n \xi_{1} & -\sigma+\xi_{2} & \ddots & \ddots & 0 & \vdots \\
0 & \ddots & \ddots & \ddots & \ddots & 0 \\
0 & 0 & \ddots & \ddots & \ddots & 0 \\
\vdots & 0 & 0 & \ddots & \ddots & n(n-1) \lambda+n \lambda \gamma \\
0 & \cdots & \cdots & 0 & (2-2 n)-\xi_{1} & n(n-1) \xi_{3}+n \xi_{2}-\sigma
\end{array}\right)\left(\begin{array}{c}
a_{0} \\
a_{1} \\
\vdots \\
\vdots \\
a_{n-1} \\
a_{n}
\end{array}\right)=0
$$

where

$$
\begin{aligned}
& \xi_{1}=(\gamma+\delta+\varepsilon), \\
& \xi_{2}=-(\lambda \gamma+\gamma+\lambda \delta+\varepsilon), \\
& \xi_{3}=-(\lambda+1) .
\end{aligned}
$$

For a nontrivial solution to exist, the determinant of the coefficients matrix must vanish which results in certain

$$
\begin{aligned}
\psi_{n}(x)= & (\cosh (x))^{(1 / 2)\left(1+\sqrt{1-4 v_{1}}\right)} \\
& \cdot\left(1+g \cosh ^{2}(x)\right)^{(1 / 2)\left(1-\sqrt{1+v_{3} /(1+g)}\right)} \\
& \cdot \sum_{m=0}^{n} a_{m}\left(-\sinh ^{2}(x)\right)^{m},
\end{aligned}
$$
three-term recurrence relation:
As can be seen, this relation is the same as the energy relation of the BAM given in (10). Hence, as a result of (24), the operator $H$ preserves the $(n+1)$-dimensional invariant subspace $\phi(z)=\sum_{m=0}^{n} a_{m} z^{m}$ spanned by the basis $\left\langle 1, z, z^{2}, \ldots, z^{n}\right\rangle$ and thereby using the representation theory of $s l(2)$ we can determine the solutions of the first $n+1$ states exactly. Accordingly, (24) can be represented as the following matrix equation:

relations between the potential parameters, which will be discussed in detail later. Also, from (4), the wave function is as

where the expansion coefficients $a_{m}$ satisfy the following

$$
a_{m}=\frac{-\left((m-1)(m-2) \xi_{3}+(m-1) \xi_{2}-\sigma\right) a_{m-1}-\left((6-6 m)-3 \xi_{1}\right) a_{m-2}}{(m(m-1) \lambda+m \lambda \gamma)}
$$


with boundary conditions $a_{-1}=a_{-2}=0$ and $a_{n+1}=0$. Therefore, we have succeeded in finding the general exact expressions for the energy, wave function, and the relation between the potential parameters. In other words, we can quickly obtain the exact solutions of the QES DWP for any arbitrary $n$ from (25), (26), and (28). In the following, in order to explain the method in more detail and also to compare these results with those obtained by the BAM in the previous section, we study the first three states. For $n=0$, from (25), the energy equation is given by

$$
\frac{1}{16}\left(3+\sqrt{1-4 v_{1}}-2 \sqrt{1+\frac{v_{3}}{1+g}}\right)^{2}+\frac{E}{4}=0
$$

and the restriction on the potential parameters is obtained from (26) as

$$
\begin{aligned}
& \frac{\lambda}{4}\left(\frac{1}{2}+\frac{1}{2} \sqrt{1-4 v_{1}}+\frac{1}{\lambda}-\frac{1}{\lambda} \sqrt{1+\frac{v_{3}}{1+g}}-v_{1}-\frac{v_{2}}{g \lambda}\right. \\
& \left.-\frac{v_{3}}{g^{2} \lambda^{2}}+E\right)=0 .
\end{aligned}
$$

The energy eigenvalues of the first excited state associated with $n=1$ are given as

$$
\begin{aligned}
& \frac{1}{16}\left(3+\sqrt{1-4 v_{1}}-2 \sqrt{1+\frac{v_{3}}{1+g}}\right)^{2}+\frac{E}{4} \\
& \quad=-(\gamma+\delta+\varepsilon),
\end{aligned}
$$

where the potential parameters satisfy the constraint

$$
\begin{aligned}
& \left(-300 E+300 v_{1}+240 v_{2}+192 v_{3}-250 \sqrt{1-4 v_{1}}\right. \\
& -970) \sqrt{25+20 v_{3}}+\left(3750 E-3750 v_{1}-3000 v_{2}\right. \\
& \left.-2400 v_{3}+8125\right) \sqrt{1-4 v_{1}}+\left(-2500 E+2000 v_{2}\right. \\
& \left.+1600 v_{3}-17000\right) v_{1}+\left(-2000 E+1280 v_{3}\right. \\
& -8600) v_{2}+(-1600 E-6680) v_{3}+1250 E^{2} \\
& +10750 E+13725+1250 v_{1}^{2}+800 v_{2}^{2}+512 v_{3}^{2} \\
& =0 .
\end{aligned}
$$

Likewise, for the second excited state $n=2$, we have

$$
\begin{aligned}
& \frac{1}{16}\left(3+\sqrt{1-4 v_{1}}-2 \sqrt{1+\frac{v_{3}}{1+g}}\right)^{2}+\frac{E}{4} \\
& \quad=-2(\gamma+\delta+\varepsilon)-2,
\end{aligned}
$$

with the following constraints on the potential parameters:

$$
\begin{aligned}
& -\frac{125}{64} E^{3}+\left(\frac{375}{64} v_{1}-\frac{8325}{128}+\frac{75}{64} \sqrt{25+20 v_{3}}+\frac{75}{16} v_{2}\right. \\
& \left.+\frac{15}{4} v_{3}-\frac{1875}{128} \sqrt{1-4 v_{1}}\right) E^{2}+\frac{E}{16000}\left(-150000 v_{2}\right. \\
& \left.-120000 v_{3}+3925000\right) v_{1}+\frac{E}{16000}\left(375000 v_{2}\right. \\
& \left.+468750 v_{1}+300000 v_{3}-3903125\right) \sqrt{1-4 v_{1}} \\
& +\frac{E}{16000}\left(-30000 v_{2}-37500 v_{1}-24000 v_{3}\right. \\
& \left.+376250+73750 \sqrt{1-4 v_{1}}\right) \sqrt{25+20 v_{3}}+\left(-\frac{15}{4}\right. \\
& \cdot v_{2}^{2}+\frac{1}{16000}\left(-96000 v_{3}+1665000\right) v_{2}-\frac{375}{64} v_{1}^{2} \\
& \left.+\frac{1273}{16} v_{3}-\frac{12}{5} v_{3}^{2}-\frac{72365}{128}\right) E-\frac{32895}{32} \\
& +\frac{1}{16000}\left(30720 v_{3}^{2}-1018400 v_{3}+7236500\right) v_{2} \\
& +\frac{\sqrt{25+20 v_{3}}}{16000}\left(\left(426625-73750 v_{1}-59000 v_{2}\right.\right. \\
& \left.\left.-47200 v_{3}\right) \sqrt{1-4 v_{1}}+12000 v_{2}^{2}+18750 v_{1}^{2}\right) \\
& +\frac{1}{16000}\left(\left(30000 v_{2}+24000 v_{3}-545000\right) v_{1}\right. \\
& \left.+7680 v_{3}^{2}-239000 v_{3}\right) \sqrt{25+20 v_{3}} \\
& +\frac{1}{16000}\left(\left(19200 v_{3}-301000\right) v_{2}+1174625\right) \\
& \cdot \sqrt{25+20 v_{3}}+\frac{1}{16000}\left(-234375 v_{1}^{2}\right. \\
& +\left(-375000 v_{2}-300000 v_{3}+4606250\right) v_{1} \\
& \left.-96000 v_{3}^{2}\right) \sqrt{1-4 v_{1}}+\frac{1}{16000}\left(-150000 v_{2}^{2}\right. \\
& +\left(-240000 v_{3}+3122500\right) v_{2}+2430500 v_{3} \\
& -9027500) \sqrt{1-4 v_{1}}+\frac{53711}{160} v_{3}+\frac{64}{125} v_{3}{ }^{3}+\frac{125}{64} \\
& \cdot v_{1}^{3}+\frac{1}{16000}\left(75000 v_{2}+60000 v_{3}-2884375\right) v_{1}^{2} \\
& +v_{2}^{3}+\frac{1}{16000}\left(60000 v_{2}^{2}+\left(96000 v_{3}-3140000\right)\right. \\
& \left.\cdot v_{2}+38400 v_{3}^{2}-2453000 v_{3}+17311250\right) v_{1} \\
& -\frac{607}{25} v_{3}^{2}+\frac{1}{16000}\left(38400 v_{3}-666000\right) v_{2}^{2}=0 \text {. }
\end{aligned}
$$

Our numerical results for the first four states are reported and compared in Table 1. 


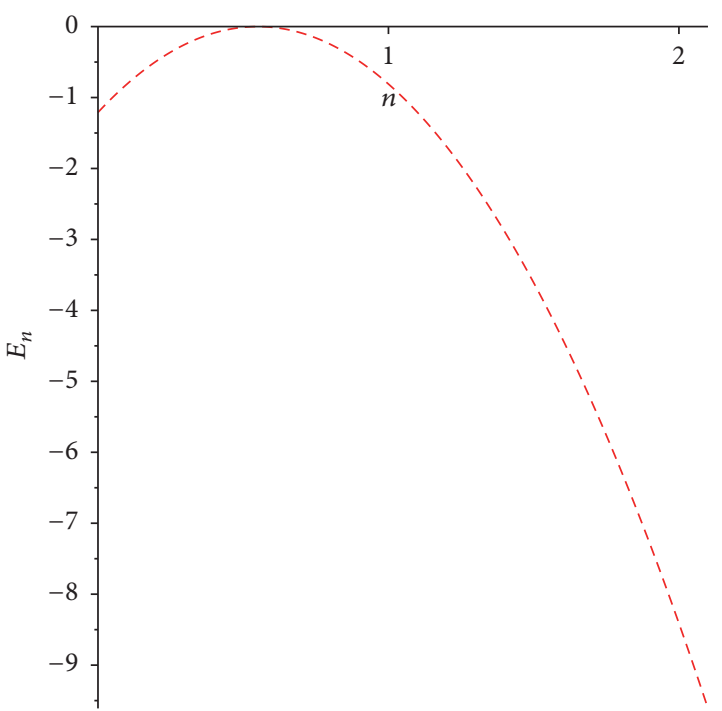

FIGURE 1: The variations of energy versus $n$ with $v_{1}=0.09, v_{3}=10$, and $g=1 / 4$.

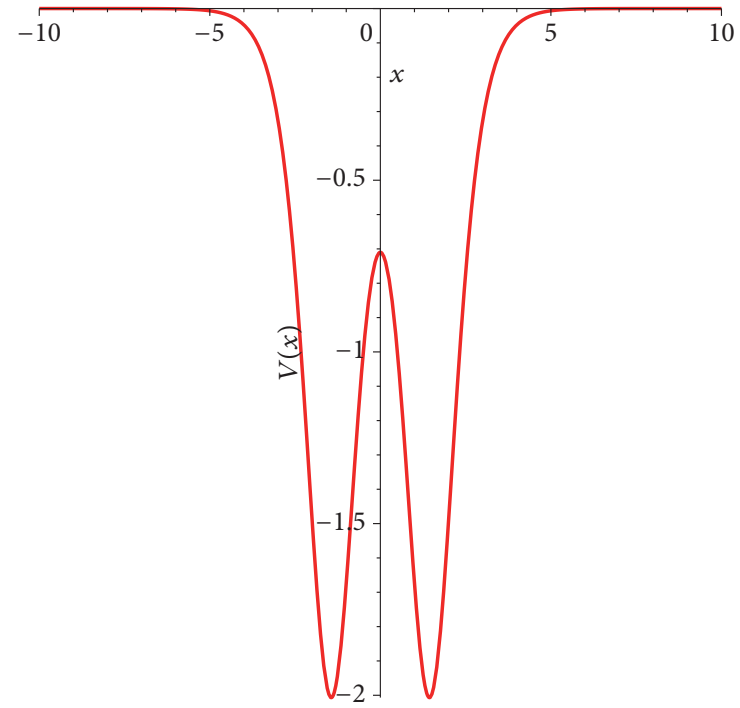

FIgure 2: Plot of the QES DWP as a function of $x$ for $v_{1}=0.09$, $v_{2}=-9$, and $v_{3}=10$.

\section{Discussion and Numerical Results}

As shown in (11) and (26), the three potential parameters $v_{1}$, $v_{2}$, and $v_{3}$ are not independent of one another; rather they are connected by certain relations. For the present problem, from (10), the parameters $v_{1}$ and $v_{3}$ must preserve the following conditions:

$$
\begin{aligned}
& v_{1}<\frac{1}{4}, \\
& v_{3}>-(1+g),
\end{aligned}
$$

to obtain real energy eigenvalues. The third parameter, $v_{2}$, can then be determined from (26). In the following, some of our numerical results for the energy eigenvalues and the allowed values of potential parameters are reported and compared in Table 1. For comparison purpose, we use the parameter set given in [17]. It is observed that our results are identical with those obtained in [17] via a different method. Also, in Figure 1, the variations of energy as a function of $n$ are plotted for the allowed values of the potential parameters.

\section{Conclusions}

Using the Bethe ansatz method, we have solved the Schrödinger equation for a new QES DWP and obtained the general exact expressions for the energies and the corresponding wave functions as well as the allowed values of the potential parameters in terms of the roots of the Bethe ansatz equations. In addition, we have solved the same problem using the Lie algebraic approach within the framework of quasi-exact solvability and obtained the exact solutions using the representation theory of $s l(2)$ Lie algebra. Also, we have reported some numerical results and shown that the results are in good agreement with each other and with those obtained previously by using a different method.

\section{Competing Interests}

The authors declare that they have no competing interests.

\section{References}

[1] L. Infeld and T. E. Hull, “The factorization method," Reviews of Modern Physics, vol. 23, pp. 21-68, 1951

[2] F. Cooper, A. Khare, and U. Sukhatme, "Supersymmetry and quantum mechanics," Physics Reports, vol. 251, no. 5-6, pp. 267385, 1995.

[3] S.-H. Dong, Factorization Method in Quantum Mechanics, vol. 150 of Fundamental Theories of Physics, Springer, Dordrecht, Netherlands, 2007.

[4] S.-H. Dong, Wave Equations in Higher Dimensions, Springer, New York, NY, USA, 2011.

[5] S.-H. Dong, "Exact Solutions of the two-dimensional Schrödinger equation with certain central potentials," International Journal of Theoretical Physics, vol. 39, no. 4, pp. 1119-1128, 2000.

[6] J. Bougie, A. Gangopadhyaya, and J. V. Mallow, "Generation of a complete set of additive shape-invariant potentials from an Euler equation," Physical Review Letters, vol. 105, no. 21, Article ID 210402, 2010.

[7] J. Bougie, A. Gangopadhyaya, J. Mallow, and C. Rasinariu, "Supersymmetric quantum mechanics and solvable models," Symmetry, vol. 4, no. 3, pp. 452-473, 2012.

[8] J. Bougie, A. Gangopadhyaya, J. V. Mallow, and C. Rasinariu, "Generation of a novel exactly solvable potential," Physics Letters. A, vol. 379, no. 37, pp. 2180-2183, 2015.

[9] M. A. Shifman, "New findings in quantum mechanics (partial algebraization of the spectral problem)," International Journal of Modern Physics A, vol. 4, no. 12, pp. 2897-2952, 1989.

[10] A. González-López, N. Kamran, and P. J. Olver, "Normalizability of one-dimensional quasi-exactly solvable Schrodinger operators," Communications in Mathematical Physics, vol. 153, no. 1, pp. 117-146, 1993. 
[11] A. Turbiner, "Lie-algebras and linear operators with invariant subspaces," in Lie Algebras, Cohomology, and New Applications to Quantum Mechanics, vol. 160 of Contemporary Mathematics, pp. 263-310, American Mathematical Society, Providence, RI, USA, 1994.

[12] A. G. Ushveridze, Quasi-Exactly Solvable Models in Quantum Mechanics, Institute of Physics Publishing, Bristol, UK, 1994.

[13] D. Gómez-Ullate, A. González-López, and M. A. Rodríguez, "Quasi-exactly solvable generalizations of calogero-sutherland models," Theoretical and Mathematical Physics, vol. 127, no. 3, pp. 719-728, 2001.

[14] C.-M. Chiang and C.-L. Ho, "Charged particles in external fields as physical examples of quasi-exactly-solvable models: a unified treatment," Physical Review A, vol. 63, no. 6, Article ID 062105, 2001.

[15] Y.-Z. Zhang, "Exact polynomial solutions of second order differential equations and their applications," Journal of Physics. A. Mathematical and Theoretical, vol. 45, no. 6, Article ID 065206, 2012.

[16] Q.-T. Xie, "New quasi-exactly solvable double-well potentials," Journal of Physics. A. Mathematical and Theoretical, vol. 45, no. 17, Article ID 175302, 2012.

[17] B. Chen, Y. Wu, and Q. Xie, "Heun functions and quasiexactly solvable double-well potentials," Journal of Physics A: Mathematical and Theoretical, vol. 46, no. 3, 2013.

[18] H. Panahi and M. Baradaran, "Unified treatment of a class of spherically symmetric potentials: quasi-exact solution," Advances in High Energy Physics, vol. 2016, Article ID 8710604, 12 pages, 2016.

[19] A. Schulze-Halberg, "Quasi-exact solvability of a hyperbolic intermolecular potential induced by an effective mass step," International Journal of Mathematics and Mathematical Sciences, vol. 2011, Article ID 358198, 9 pages, 2011.

[20] R. Koç, E. Olğar, and H. Mutaf, "A novel approach to solve quasiexactly solvable pauli equation," Advances in Mathematical Physics, vol. 2015, Article ID 304093, 5 pages, 2015.

[21] A. Gangopadhyaya, A. Khare, and U. P. Sukhatme, "Methods for generating quasi-exactly solvable potentials," Physics Letters A, vol. 208, no. 4-6, pp. 261-268, 1995.

[22] T. Wu, "Characteristic Values of the two minima problem and quantum defects of $f$ states of heavy atoms," Physical Review, vol. 44, no. 9, pp. 727-731, 1933.

[23] C. M. Bender and G. V. Dunne, "Quasi-exactly solvable systems and orthogonal polynomials," Journal of Mathematical Physics, vol. 37, no. 1, pp. 6-11, 1996.

[24] M. Razavy, "An exactly soluble Schrodinger equation with a bistable potential," American Journal of Physics, vol. 48, no. 4, pp. 285-288, 1980.

[25] H. Hassanabadi, S. F. Forouhandeh, H. Rahimov, S. Zarrinkamar, and B. H. Yazarloo, "Duffin-Kemmer-Petiau equation under a scalar and vector Hulthen potential; an ansatz solution to the corresponding Heun equation," Canadian Journal of Physics, vol. 90, no. 3, pp. 299-304, 2012.

[26] S. Hassanabadi, A. A. Rajabi, and S. Zarrinkamar, "Cornell and kratzer potentials within the semirelativistic treatment," Modern Physics Letters A, vol. 27, no. 10, Article ID 1250057, 7 pages, 2012.

[27] H. Sobhani and H. Hassanabadi, "Study of Davydov-Chaban approach considering shifted Killingbeck potential for any lstate," Modern Physics Letters A, vol. 31, no. 27, Article ID 1650152, 2016.
[28] S. H. Dong, "On the solutions of the Schrödinger equation with some Anharmonic potentials: wave function Ansatz," Physica Scripta, vol. 65, no. 4, p. 289, 2002.

[29] S. Dong, "A new approach to the relativistic Schrödinger equation with central potential: ansatz method," International Journal of Theoretical Physics, vol. 40, no. 2, pp. 559-567, 2001.

[30] M. F. Manning, "Energy levels of a symmetrical double minima problem with applications to the $\mathrm{NH}_{3}$ and $\mathrm{ND}_{3}$ molecules," Journal of Chemical Physics, vol. 3, no. 3, pp. 136-138, 1935. 

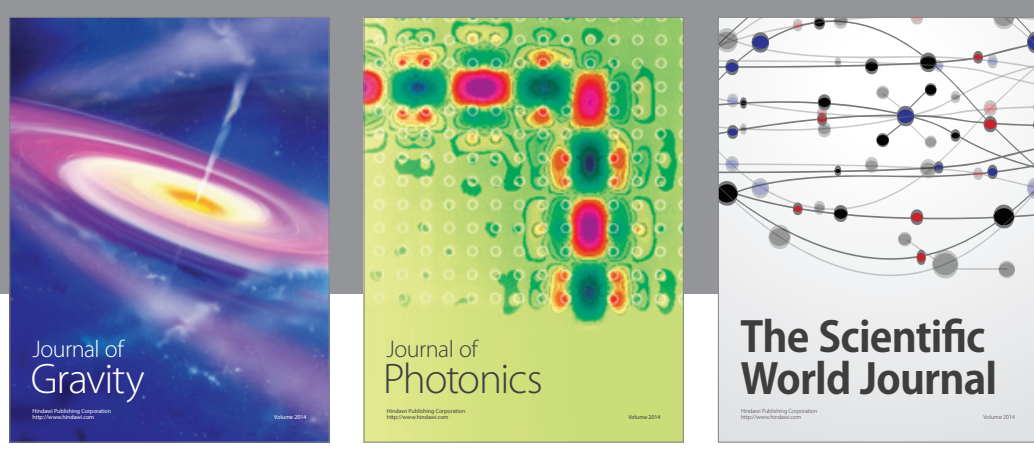

The Scientific World Journal
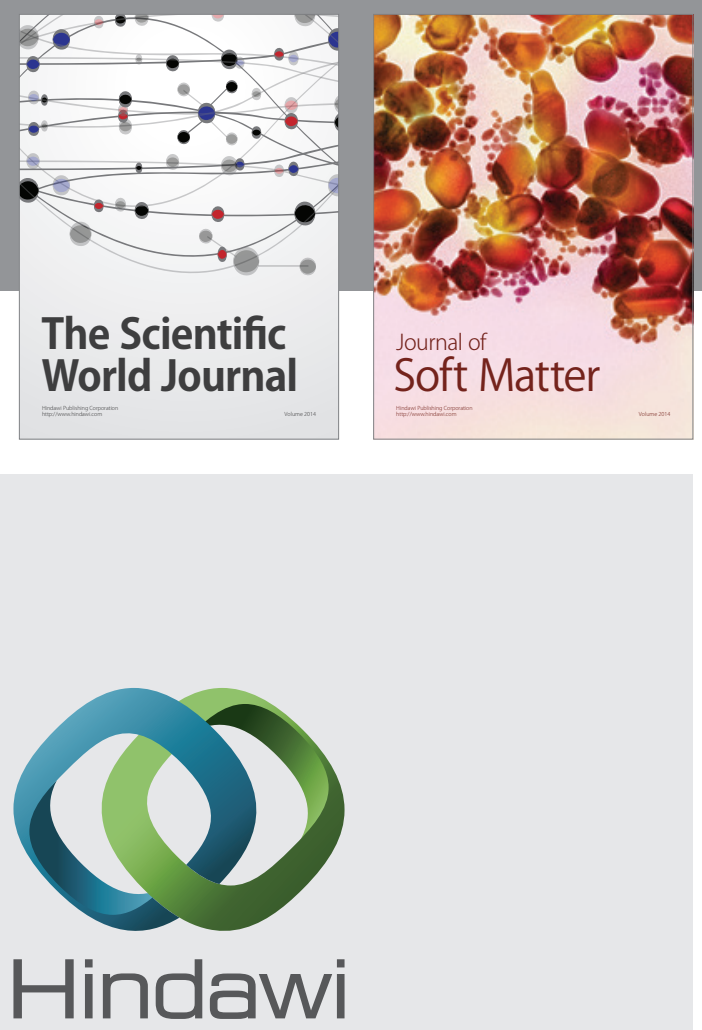

Submit your manuscripts at

https://www.hindawi.com
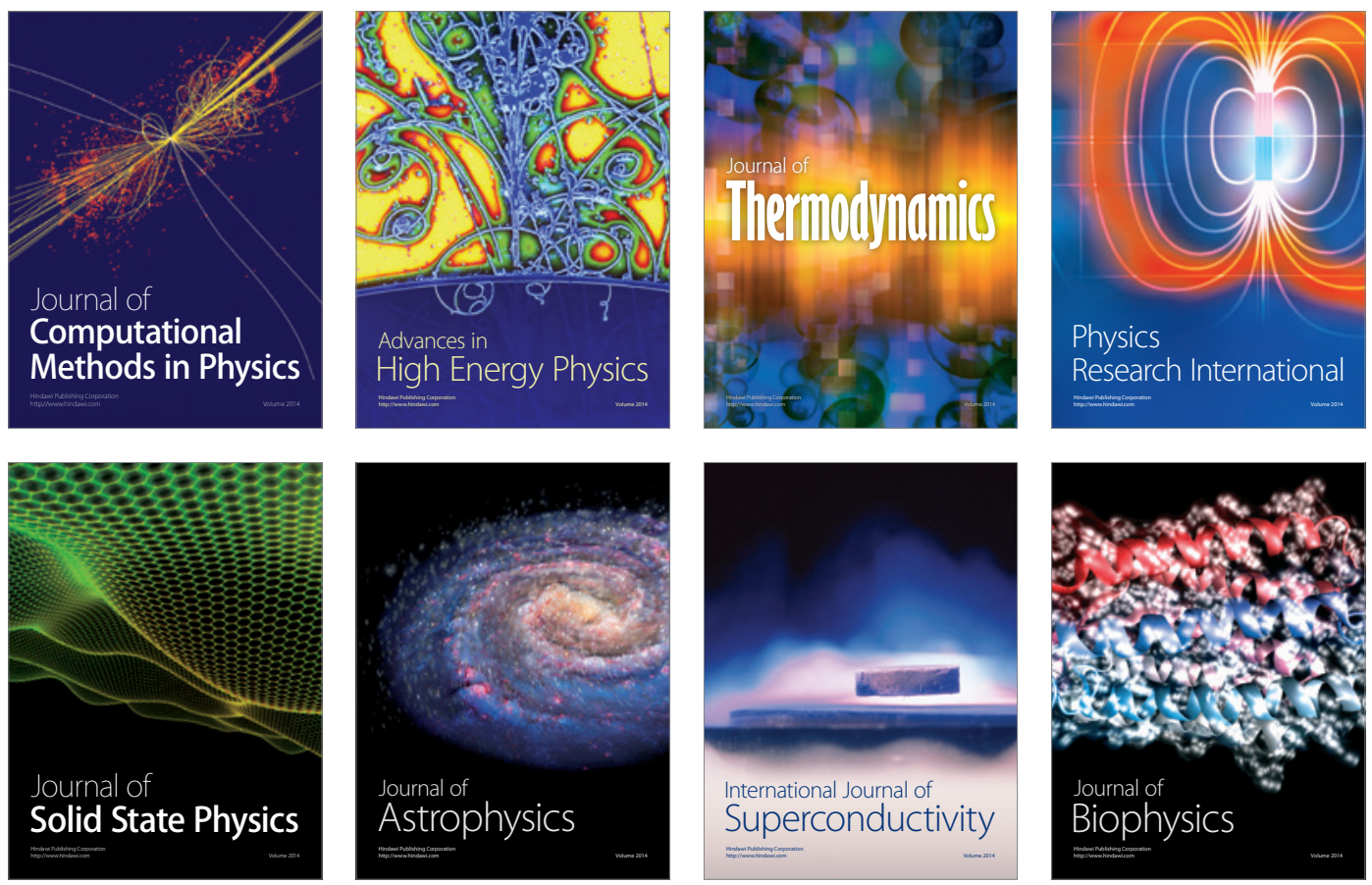
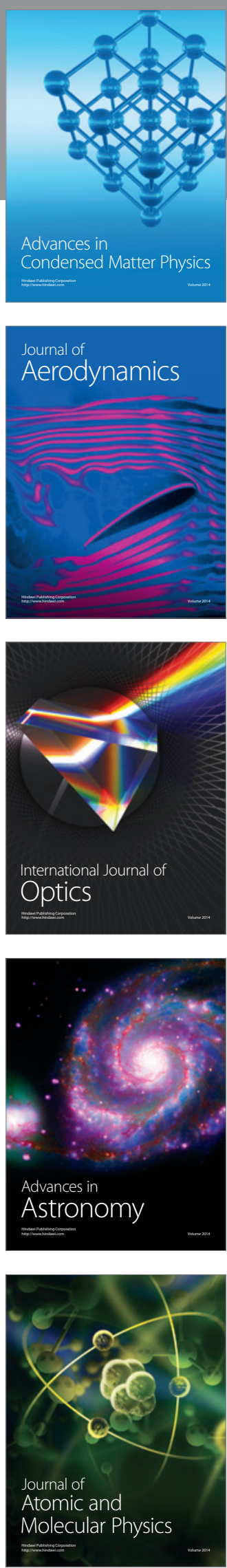